\title{
Níveis de infestação de Zaprionus indianus (Diptera: Drosophilidae) em diferentes frutos hospedeiros
}

\author{
Infestation levels of Zaprionus indianus (Diptera: Drosophilidae) in different host fruits \\ Júlia Gabriela Aleixo Vieira*, Jutiane Wollmann, Daniele Cristine Hoffmann Schlesener, Sávio Ritta \\ Mendes e Flávio Roberto Mello Garcia
}

Universidade Federal de Pelotas, Pelotas, RS, Brasil. *Autor para correspondência: ju-aleixo@hotmail.com.

Submissão: 07/07/2018 / Aceite: 25/02/2019

\begin{abstract}
RESUMO
A mosca-do-figo, Zaprionus indianus Gupta, 1970 (Diptera: Drosophilidae) é uma espécie generalista, mas importante economicamente na cultura do figo, onde é considerada praga primária. Esse estudo teve como objetivo verificar os níveis de infestação natural de $Z$. indianus em frutos de amora, araçá, fisális, goiaba, mirtilo, morango e pitanga na região sul do Brasil. Os índices foram calculados por meio do número de moscas emergidas por fruto e do número de moscas por massa $(\mathrm{kg})$ de fruto. Os maiores índices de infestação (mosca/kg) foram verificados em amoras (1295), araçás (1253) e pitangas (1016), enquanto que para o segundo índice $(M / n)$ os maiores índices foram verificados em goiabas $(26,17)$, araçás $(11,87)$ e morangos $(10,27)$. Frutos coletados diretamente da planta foram mais infestados em relação a frutos coletados no solo, o que pode ser devido ao ataque inicial de Drosophila suzukii (Matsumura, 1931) (Diptera: Drosophilidae) e Anastrepha spp., pragas já disseminadas na região.
\end{abstract}

PALAVRAS-CHAVE: Drosophilidae, espécie invasora, mosca-do-figo, praga.

\begin{abstract}
The fig fly, Zaprionus indianus Gupta, 1970 (Diptera: Drosophilidae) is a generalist species, but economically important in the fig culture, in which it is considered a primary pest. This study aimed to verify the levels of natural infestation of Z. indianus in blackberry, strawberry guava, Physalis, guava, blueberry, strawberry and Surinam cherry fruits in southern Brazil. The indexes were calculated through the number of flies emerged per fruit and the number of flies per mass $(\mathrm{Kg})$ of fruit. The highest rates of infestation (fly/Kg) were observed in blackberries (1295), strawberry guavas (1253) and Surinam cherries (1016); for the second index, $(\mathrm{M} / \mathrm{n})$ in guavas (26.17), strawberry guavas (11.87) and strawberries (10.27). Fruits collected directly from the plant were more infested compared with fruits collected from the soil, which may be due to the initial attack of Drosophila suzukii (Matsumura, 1931) (Diptera: Drosophilidae) and Anastrepha spp., pests already disseminated in the region.
\end{abstract}

KEYWORDS: Drosophilidae, invasive species, fig-fly, pest.

A mosca-do-figo, Zaprionus indianus Gupta, 1970 (Diptera: Drosophilidae) é uma espécie invasora com alto potencial de colonização, elevada ocorrência em locais antropizados e considerada uma das espécies mais abundantes entre a comunidade de drosofilídeos no Brasil (SILVA et al. 2005). Possui aproximadamente três milímetros, olhos vermelhos e coloração amarelada, sendo facilmente identificada pela presença de três espinhos no fêmur da perna anterior, e pela presença de listras brancas longitudinais intercaladas por listras pretas estreitas na região da cabeça e tórax (VAN DER LINDE 2010).

A intensificação do comércio mundial de frutos foi provavelmente a principal forma de entrada da espécie no Brasil (STEIN et al. 2003). Em pouco tempo assumiu o status de praga primária para o figo, Ficus carica L. var. Roxo de Valinhos (Rosales: Moraceae), devido a capacidade de depositar ovos no interior do ostíolo do fruto, quando este ainda se encontra em início de amadurecimento (STEIN et al. 2003). As larvas eclodem e penetram no fruto utilizando-o como sítio de desenvolvimento e atraindo outros indivíduos da espécie, que intensificam os danos e inviabilizam o consumo in natura dos frutos (PASINI \& LÚCIO 2014, GOMES et al. 2003). 
A primeira ocorrência de Z. indianus no Brasil foi registrada em 1999 em São Paulo, em caquis (Diospyros kaki) (VILELA 1999). Após sua introdução, a espécie rapidamente se disseminou pelo território brasileiro e encontra-se em diversos Estados do país (CASTRO \& VALENTE 2001, DE TONI et al. 2001, SANTOS et al. 2003, TIDON et al. 2003, KATO et al. 2004, LEÃO \& TIDON 2004, LOH \& BITNER-MATHÉ 2005, FERNANDES \& ARAÚJO 2011, BARBOSA et al. 2012, VASCONCELOS et al. 2017). Os registros também incluem outros países da América do Sul, como Uruguai (GÕNI et al. 2001) e Argentina (SOTO et al. 2006).

Zaprionus indianus possui alto potencial biótico, ciclo biológico curto e hábito generalista (VILELA \& ZUCCHI 2015). É considerada praga secundária de diversos frutos em estágio avançado de amadurecimento ou previamente danificados podendo utilizar os mesmos como sítio de oviposição e desenvolvimento (VAN DER LINDE 2010, COMMAR et al. 2012). O conhecimento de hospedeiros alternativos de espécies com status de praga é necessário para o desenvolvimento de programas de manejo, pois estes podem permitir a sobrevivência e a reprodução da espécie, principalmente quando seu hospedeiro preferencial não se encontra disponível (LEE et al. 2015).

Tendo em vista a capacidade de adaptação de $Z$. indianus em diferentes hospedeiros em regiões temperadas, 0 objetivo desse estudo foi conhecer espécies frutícolas que podem servir para 0 desenvolvimento e permanência dessa espécie no ambiente agrícola, e determinar os índices de infestação em frutos cultivados em três áreas em uma região no sul do Rio Grande do Sul, que se destaca na produção de frutas de clima temperado no Brasil.

Os frutos foram coletados semanalmente durante setembro de 2015 a agosto de 2016. As áreas de

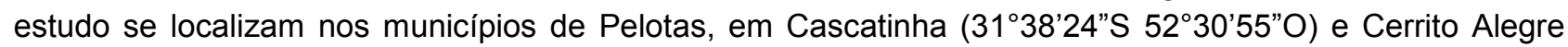
$\left(31^{\circ} 35^{\prime} 12^{\prime \prime} S 52^{\circ} 25^{\prime} 35^{\prime \prime} \mathrm{O}\right)$, e no município de Morro Redondo, em Rincão da Caneleira (313' 37 88"S $52^{\circ} 34^{\prime} 23$ 92"O), no sul do Rio Grande do Sul.

Na propriedade de Cascatinha foram amostrados frutos de amoreira-preta (Rubus spp.), araçazeiro amarelo e vermelho (Psidium cattleianum Sabine) e pitangueira (Eugenia uniflora L.). Na propriedade de Cerrito Alegre foram coletados frutos de morangueiro (Fragaria $x$ ananassa), enquanto que na propriedade no Rincão da Caneleira foram coletados amoreira-preta (Rubus spp.) e mirtileiro (Vaccinium myrtillus L.). Também foram coletados frutos de espécies que ocorrem de forma esporádica nos arredores dos pomares, como frutos de fisális (Physalis spp.), cerejeira-do-mato (Eugenia involucrata DC.) e goiabeira (Psidium guajava L.).

Os frutos foram coletados de forma aleatório da planta e do solo, conforme quantidade disponível e época de frutificação das espécies frutícolas (GARCIA \& NORRBOM 2011). Os frutos foram acondicionados em caixa térmica e encaminhados ao laboratório, onde foram pesados, identificados e acondicionados individualmente em recipientes plásticos $(250 \mathrm{~mL})$ sobre uma fina camada de vermiculita $(1 \mathrm{~cm})$, e tampados com tampa perfurada (2 cm de diâmetro) coberta por tecido voile para possibilitar as trocas gasosas. Os recipientes contendo os frutos foram acondicionados em sala climatizada [temperatura de $23 \pm 2{ }^{\circ} \mathrm{C}, 70 \%$ de umidade relativa (UR) e $12: 12 \mathrm{~h}$ de fotoperíodo], onde permaneceram até a emergência dos adultos. Os adultos foram retirados dos recipientes e acondicionados em microtubos $(1,5 \mathrm{~mL})$ contendo álcool 70\%. Posteriormente, o material foi analisado com o auxílio de estereomicróscopio, e a identificação e sexagem de $Z$. indianus foi realizada segundo caracteres morfológicos específicos (VAN DER LINDE 2010).

$O$ índice de infestação de $Z$. indianus foi determinado por amostragem, através de duas equações: (1) (M/PF), onde $M$ = número de moscas emergidas e $P F=$ peso dos frutos; e (2) $(M / n)$, onde $M$ = número de moscas emergidas e $n=$ total de frutos infestados (GARCIA \& NORRBOM 2011).

Foram amostrados 3.205 frutos, sendo 2.901 frutos coletados das plantas e 304 frutos do solo (Figura 1). Não foram coletados frutos de amora, goiaba, mirtilo e morango do solo, em função de não haver frutos caídos no chão. À exceção de fisális e cereja-do-mato, todos os frutos das espécies botânicas coletadas serviram como hospedeiros de $Z$. indianus.

Percentualmente, os frutos mais infestados foram araçás coletados diretamente do solo (56\%) e de goiabas $(42,86 \%)$, e araçás $(32,4 \%)$ retirados da planta (Figura 2). Os frutos menos infestados foram amoras $(12,6 \%)$, pitangas retiradas das plantas (9\%), morangos (8,2\%), pitangas coletadas do solo $(2,5 \%)$ e mirtilos (0,6\%) (Figura 2).

Os maiores índices de infestação relacionando o número de moscas emergidas por quilo de fruto infestado (M/PF) foram obtidos em frutos de amora (1295), seguido por araçás (1253) e pitanga (1016) coletados das plantas (Figura 3). Apesar de Z. indianus preferir frutos maduros/em apodrecimento ou com danos devido à queda (PASINI \& LÚCIO 2014), houve menor índice de infestação em frutos coletados no solo, como araçás (997) e pitangas (1000) (Figura 3). Esse fato pode estar relacionado com a menor 
amostragem em solo, uma vez que os frutos caídos eram constantemente recolhidos para evitar a proliferação de pragas. Outra hipótese é a capacidade de postura na epiderme dos frutos, e após a eclosão as larvas penetram nos frutos saudáveis (BERNARDI et al. 2017). A presença de Drosophila suzukii (Matsumura, 1931) (Diptera: Drosophilidae), Anastrepha fraterculus (Wiedemann, 1830) (Diptera: Tephritidae) e besouros da família Nitidulidae causam danos prévios que servem como porta de entrada para $Z$. indianus, visto que, essa espécie não é capaz de perfurar frutos íntegros para depositar ovos, sendo assim necessária a presença de um dano inicial provocado por outro organismo (STECK 2005, JOSHI et al. 2014).

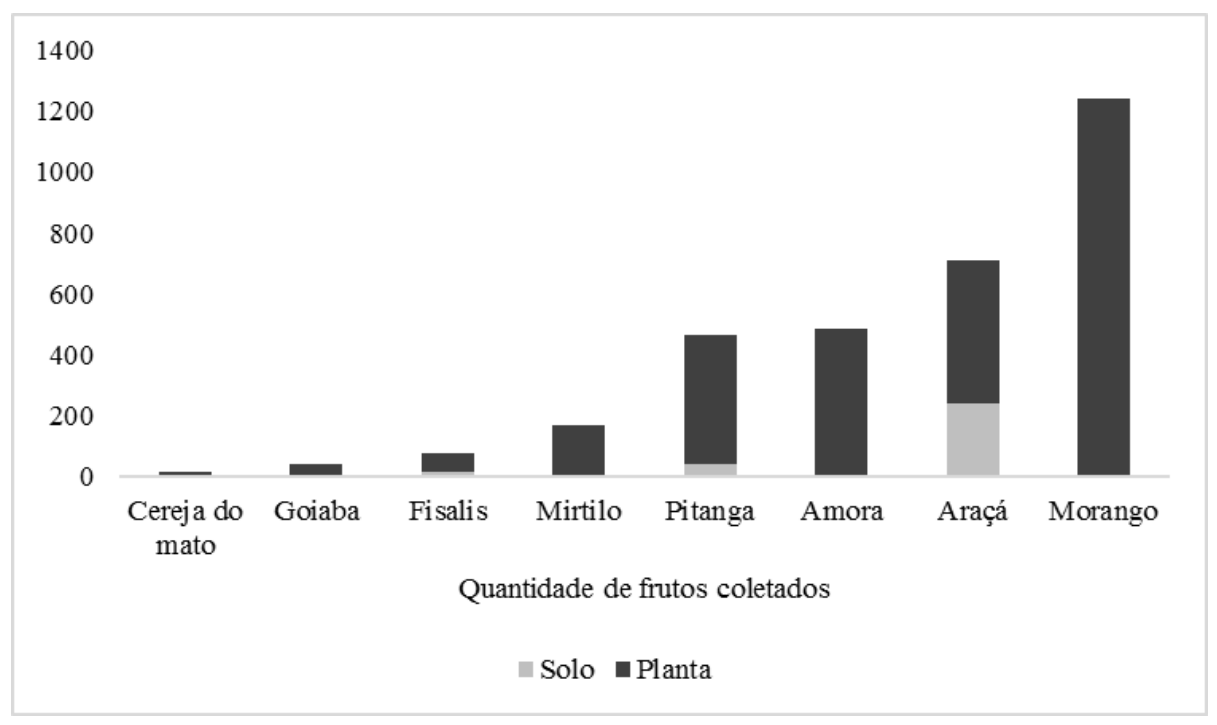

Figura 1. Número de frutos coletados da planta e do solo.

Figure 1. Number of fruits collected from the plant and soil.

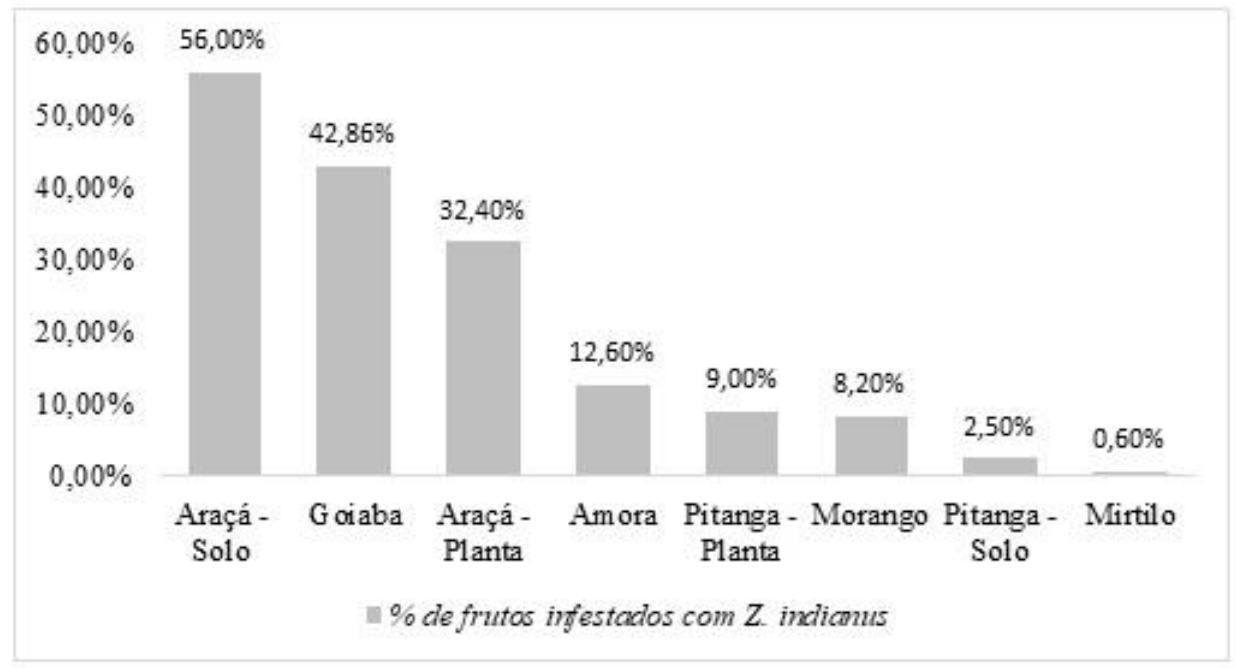

Figura 2. Percentual (\%) de frutos infestados por Zaprionus indianus.

Figure 2 Percentage (\%) of fruits infested by Zaprionus indianus.

Em relação ao índice do número de moscas emergidas pelo total de frutos infestados $(\mathrm{M} / \mathrm{n})$, o maior valor foi registrado em goiabas $(26,17)$, seguido por araçás $(11,87)$, morangos $(10,27)$, amoras $(8,92)$, pitangas $(4,82)$ e mirtilos $(1,00)$ coletados das plantas (Figura 4). O maior índice observado em goiabas possivelmente se deve pelo fato do fruto apresentar tamanho médio superior aos demais frutos amostrados, podendo assim, sustentar um maior número de larvas (CARVALHO et al. 2004). Para frutos coletados no solo, o índice $(\mathrm{M} / \mathrm{n})$ mais elevado foi observado para araçás $(7,9)$ em relação a pitangas $(5,00)$ (Figura 4$)$.

Em relação as espécies associadas a $Z$. indianus, $A$. fraterculus é a praga primária mais abundante e importante em frutíferas no sul do Brasil, atacando frutos nas diversas estações do ano (GARCIA et al. 2003). Enquanto que, D. suzukii, foi registrada em 2013 na região sul do Brasil (DEPRÁ et al. 2014) e é 
considerada uma das principais pragas em cultivos de pequenos frutos e frutos de caroço (CINI et al. 2012, SCHLESENER et al. 2017) e, assim como a mosca-das-frutas sul-americana, ataca frutos íntegros ainda na planta (ATALLAH et al. 2014, LEE et al. 2015). Espécies de besouros Nitidulidae podem atacar frutos sadios, a exemplo de Lobiopa insularis (Castelnau, 1840) (Coleoptera: Nitidulidae) (ANDZEIEWSKI et al. 2018), sendo os danos ocasionados tanto por larvas quanto por adultos, que também utilizam esse substrato para alimentação e oviposição (RONDON et al. 2011).

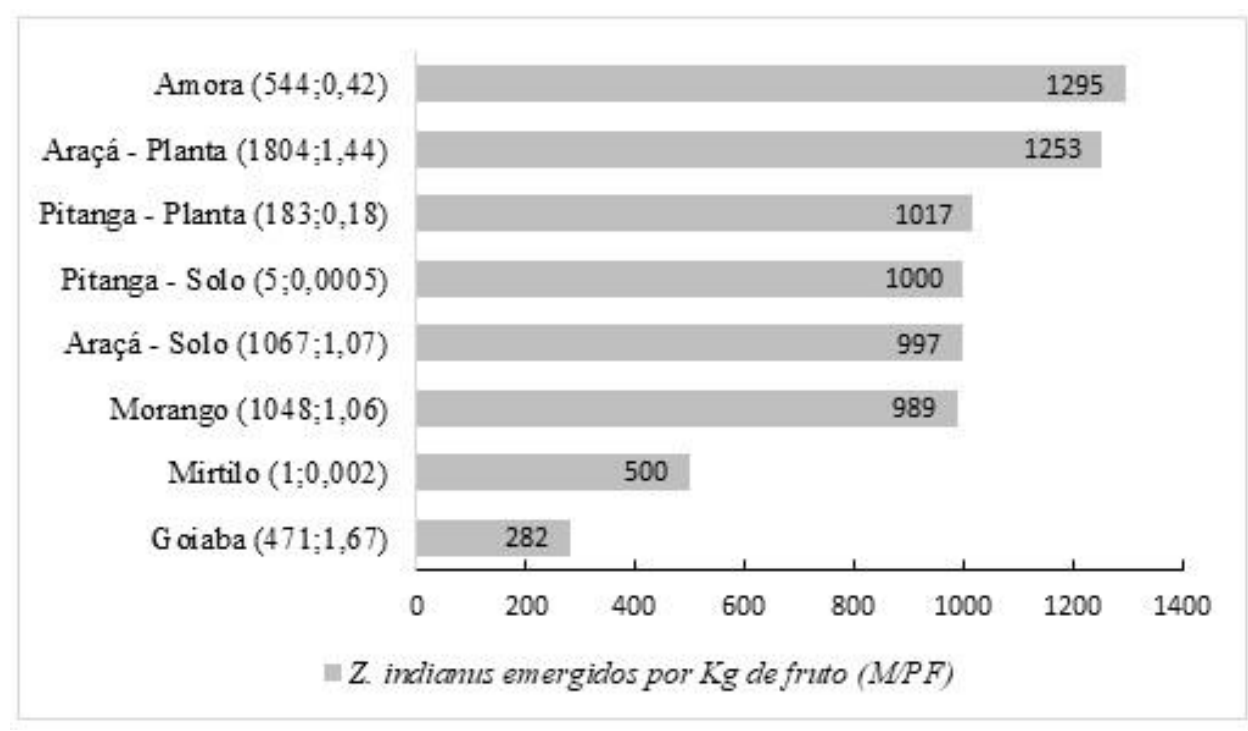

Figura 3. Número total de adultos de Zaprionus indianus emergidos por quilo de fruto infestado (M/PF). Os números entre parênteses após o nome dos frutos indicam o total de adultos emergidos da espécie vegetal e o peso total dos frutos infestados, respectivamente.

Figure 3. Total number of adults of Zaprionus indianus emerged per kilogram of infested fruit (M/PF). The numbers in brackets after the name of the fruits indicate the adults emerged from the plant species and the total weight of the infested fruits, respectively.

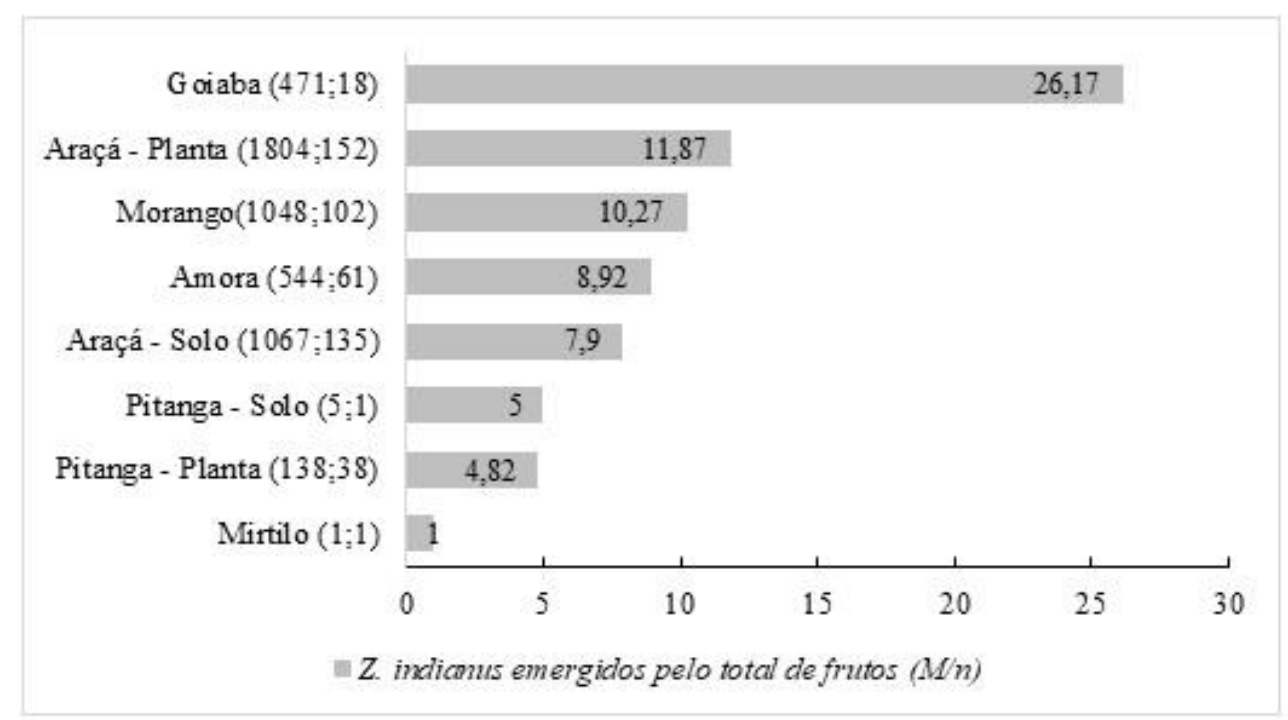

Figura 4. Número total de adultos de Zaprionus indianus emergidos/fruto ( $M / n)$. Os números entre parênteses após o nome dos frutos indicam o número total de adultos emergidos da espécie vegetal e o total de frutos infestados, respectivamente.

Figure 4. Total number of adults of Zaprionus indianus emerged per fruit (M/n). The numbers in backets after the name of the fruits indicate the total number of adults emerged from the plant species and the total number of infested fruits, respectively.

Alguns estudos relatam a associação entre $Z$. indianus e as espécies acima citadas em diversos frutos. Já foi relatada a presença da mosca-do-figo em morangos previamente danificados por $D$. suzukii 
(NAVA et al. 2015) em diferentes estágios de maturação, evidenciando o comportamento oportunista da espécie (BERNARDI et al. 2017). A ocorrência de $Z$. indianus com pragas primárias também foi observada em araçás, butiás [Butia capitata (Mart.) Beccari], morangos e tomates (Solanum lycopersicon Mill.), em que se observou a emergência de $Z$. indianus e de Anastrepha spp. (PERIPOLLI 2015) e, ainda, em frutos de goiaba coletados do chão e/ou das plantas (LASA et al. 2017). A mosca-do-figo também foi registrada em armadilhas de captura para monitoramento de $D$. suzukii no período de pré e pós colheita em cultivos de pêssego [Prunus persica (L.) Batsch], mirtilo, framboesa (Rubus idaeus L.), morango, cereja (Prunus avium L.) e ameixa (Prunus sp.), evidenciando a importância do monitoramento para ambas as espécies, tendo em vista que $Z$. indianus pode potencializar os danos em frutos já infestados por outras espécies (RENKEMA et al. 2013).

Os dados encontrados nesse estudo demonstram que frutos de amora, araçá, goiaba, mirtilo, morango e pitanga podem ser utilizados como hospedeiros para a espécie $Z$. indianus, e que a infestação pela mosca-do-figo em frutos íntegros pode ser favorecida pelo ataque de pragas primárias, aumentando as injúrias em frutos atacados e elevando o dano econômico em diversos cultivos frutícolas.

\section{REFERÊNCIAS}

ANDZEIEWSKI S et al. 2018. Thermal requirements and estimates of the annual number of generations of Lobiopa insularis on strawberry crop. Horticultura Brasileira 36: 54-58.

ATALLAH J et al. 2014. The making of a pest: the evolution of a fruit-penetrating ovipositor in Drosophila suzukii and related species. Proceedings of the Royal Society 281: 1-9.

BARBOSA MMR et al. 2012. Diptera, Drosophilidae, Zaprionus indianus Gupta 1970: distribution extension for the state of Mato Grosso do Sul, Brazil. Checklist 8: 175-176.

BERNARDI D et al. 2017. Susceptibility and interactions of Drosophila suzukii and Zaprionus indianus (Diptera: Drosophilidae) in damaging strawberry. Neotropical Entomology 46: 1-7.

CARVALHO CAL et al. 2004. Moscas-das-frutas e parasitoides associados a frutos de cajazeiras em Presidente Tancredo Neves - Bahia. Magistra 16: 85-90.

CASTRO FL \& VALENTE VLS. 2001. Zaprionus indianus is invading Drosophilid communities in the southern Brazilian city of Porto Alegre. Drosophila Information Service 84: 15-17.

CINI A et al. 2012. A review of the invasion of Drosophila suzukii in Europe and a draft research agenda for integrated pest management. Bulletin of Insectology 65: 149-160.

COMMAR LS et al. 2012. Taxonomic and evolutionary analysis of Zaprionus indianus and its colonization of Paleartic and Neotropical regions. Genetics and Molecular Biology 35: 395-406.

DEPRÁ M et al. 2014. The first records of the invasive pest Drosophila suzukii in the South American continent. Journal of Pest Science 87: 379-383.

DE TONI DC et al. 2001. First Record of Zaprionus indianus (Diptera, Drosophilidae) in the State of Santa Catarina, Brazil. Biotemas 14: 71-85.

FERNANDES DRR \& ARAÚJO EL. 2011. Ocorrência de Zaprionus indianus Gupta (Diptera: Drosophilidae) em frutos de Juazeiro Ziziphus joazeiro Mart. (Rhamnaceae) no estado do Rio Grande do Norte. Revista Brasileira de Fruticultura 33: 1356-1358.

GARCIA FRM et al. 2003. Flutuação populacional de Anastrepha fraterculus (Wiedemann, 1830) (Diptera: Tephritidae) na região oeste de Santa Catarina, Brasil. Revista Brasileira de Entomologia 47: 415-420.

GARCIA FRM \& NORRBOM AL. 2011. Tephritoid flies (Diptera, Tephritoidea) and their plant hosts from the State of Santa Catarina in Southern Brazil. Florida Entomologist 94: 151-157.

GOMES HL et al. 2003. Presence of the yeast Candida tropicalis in figs infected by the fruit fly Zaprionus indianus (Dip.: Drosophilidae). Brazilian Journal of Microbiology 34: 5-7.

GÕNI B et al. 2001. First Record of Zaprionus indianus Gupta 1970 (Diptera, Drosophilidae) in southern localities of Uruguay. Drosophila Information Service 84: 61-65.

JOSHI NK et al. 2014. First report of Zaprionus indianus (Diptera: Drosophilidae) in commercial fruits and vegetables in Pennsylvania. Journal of Insect Science 14: 1-4.

KATO CM et al. 2004. Ocorrência de Zaprionus indianus Gupta, 1970 (Diptera: Drosophilidae) no estado de Minas Gerais. Ciência e Agrotecnologia 28: 454-455.

LASA R et al. 2017. Fruit firmness, superficial damage, and location modulate infestation by Drosophila suzukii and Zaprionus indianus: the case of guava in Veracruz, Mexico. Entomologia Experimentalis et Applicata 162: 4-12.

LEÃO BFD \& TIDON R. 2004. Newly invading species exploiting nativa host-plants: the case of the african Zaprionus indianus (Gupta) in the Brazilian Cerrado (Diptera, Drosophilidae). Annales de la Société Entomologique de France 40: 285-290.

LEE JC et al. 2015. Infestation of wild and ornamental noncop fruits by Drosophila suzukii (Diptera: Drosophilidae). Annals of the Entomological Society of America 108: 117-129.

LOH R \& BITNER-MATHÉ BC. 2005. Variability of wing size and shape in three populations of a recent Brazilian invader, Zaprionus indianus (Diptera: Drosophilidae), from different habitats. Genética 125: 271-281.

NAVA DE et al. 2015. Bioecologia, monitoramento e controle de Drosophila suzukii na cultura do morangueiro. Rio 
grande do Sul: EMBRAPA. 25p. (Documento 398).

PASINI MPB \& LÚCIO AD. 2014. Ocorrência da mosca-do-figo no Rio Grande do Sul e sua emergência em diferentes frutas. Revista de Ciências Agrárias 57: 318-321.

PERIPOLLI F et al. 2015. Incidência de Zaprionus indianus Gupta, 1970 (Insecta, Drosophilidae) no noroeste do estado do Rio Grande do Sul. In: XXIII Seminário de Iniciação Cientifica. Resumos... ljuí: UNIJUÍ. 4p.

RENKEMA JM et al. 2013. First records of Zaprionus indianus Gupta (Diptera: Drosophilidae) from commercial fruit fields in Ontario and Quebec, Canada. Journal of the Entomological Society of Ontario 144: 125-130.

RONDON SI et al. 2011. Sap Beetle (Coleoptera: Nitidulidae) Management in Strawberries. Gainesville: University of Florida. 2p.

SANTOS JF et al. 2003. Colonization of Northeast Region of Brazil by the drosophilid flies Drosophila malerkotliana and Zaprionus indianus, a new potential insect pest for Brazilian fruitculture. Drosophila Information Service 86: 92-95.

SCHLESENER DCH et al. 2017. Effects of insecticides on adults and eggs of Drosophila suzukii (Diptera, Drosophilidae). Revista Colombiana de Entomologia 43: 208-214.

SILVA NM et al. 2005. Population dynamics of the invasive species Zaprionus indianus (Gupta) (Diptera: Drosophilidae) in communities of drosophilids of Porto Alegre city, southern of Brazil. Neotropical Entomology 34: 363-374.

SOTO I et al. 2006. First Record of Zaprionus indianus Gupta, 1970 (Diptera, Drosophilidae) in Argentina. Drosophila Information Service 89: 13-14.

STECK GJ. 2005. Zaprionus indianus Gupta (Diptera: Drosophilidae), a genus and species new to Florida and North America. Pest Alert: Florida. Disponível em: <http://www.freshfromflorida.com/pi/pest-alerts/zaprionus-indianus.html>. Acesso em: 02 abr. 2018.

STEIN CP et al. 2003. Aspectos biológicos da mosca do figo, Zaprionus indianus Gupta, 1970 (Diptera: Drosophilidae). Entomotropica 18: 219-221.

TIDON R et al. 2003. Impact of the colonisation of Zaprionus (Diptera, Drosophilidae) in different ecosystems of the Neotropical Region: 2 years the invasion. Biological Conservation 112: 299-305.

VASCONCELOS AS et al. 2017. Primeiro registro de Zaprionus indianus Gupta (Diptera: Drosophilidae) no estado do Acre, Brasil. EntomoBrasilis 10: 60-63.

VAN DER LINDE K. 2010. Zaprionus indianus: species identification and taxonomic position. Drosophila Information Service 93: 95-98.

VILELA CR. 1999. Is Zaprionus indianus Gupta, 1970 (Diptera, Drosophilidae) currently colonizing the Neotropical region? Drosophila Information Service 82: 37-39

VILELA EF \& ZUCCHI RA. 2015. Pragas introduzidas no Brasil: insetos e ácaros. Piracicaba: FEALQ. 908p. 